\title{
Effects of arthroscopic capsular and inferior glenohumeral ligament release on function and stability for patients with frozen shoulder
}

\section{Yao Zhang}

Sport Medicine, Northern Jiangsu People's Hospital

\section{Shichao Cao}

Sports Medicine, Norther Jiangsu People's Hospital

Mingsheng Liu

Sports Medicine Northern Jiangsu People's Hospital

Wenyong Fei

Sports Medicine, Northern jiangsu People's Hospital

Jingcheng Wang ( $\sim$ 18994874221@163.com )

https://orcid.org/0000-0003-1543-0831

Research article

Keywords: frozen shoulder, arthroscopic capsular and inferior glenohumeral ligament release, range of motion, stability of the shoulder joint

Posted Date: January 2nd, 2020

DOl: https://doi.org/10.21203/rs.2.19843/v1

License: (c) (1) This work is licensed under a Creative Commons Attribution 4.0 International License.

Read Full License 


\section{Abstract}

\section{Background}

The inferior glenohumeral ligament (IGHL) plays an important role in maintaining shoulder joint stability. However, no systematic studies on shoulder stability and function of patients with FS after IGHL release exist. This study assessed the functional and clinical outcomes of IGHL release for FS.

\section{Methods}

Forty-seven patients underwent arthroscopic capsule and IGHL release with the same postoperative rehabilitation procedure. Five functional parameters were measured postoperatively at 4, 8, 12 and 28 weeks: the apprehension test result, American Shoulder and Elbow Surgeons Score (ASES), Constant score, visual analog scale (VAS) score and active range of motion (ROM).

Results

All patients improved in the aforementioned evaluations. At 28 weeks, the ASES, Constant score and VAS score improved from $31.30 \pm 6.41$ to $92.43 \pm 3.89,30.15 \pm 6.85$ to $90.71 \pm 4.27$ and $6.73 \pm 0.72$ to $0.60 \pm 0.74$, respectively $(p<0.05)$. Forward flexion ( $F F)$ and abduction (ABD) improved from $73.85 \pm 14.94$ to $166.70 \pm 7.23$ and from $69.65 \pm 12.74$ to $165.03 \pm 6.36$, respectively $(p<0.05)$. External rotation $(E R)$ and internal rotation (IR) also significantly improved. In total, $95 \%$ of the patients were able to perform full elevation, and $97.5 \%$ of the patients could place the dorsum of their hands between their shoulder blades. All patients had negative apprehension tests, and no dislocations occurred. No other serious postoperative complications were observed.

\section{Conclusions}

Arthroscopic capsule and IGHL release was effective and could improve the active ROM and relieve pain in patients with FS. This surgical procedure did not cause instability or dislocations in the shoulder joint.

\section{Introduction}

The main clinical symptom of frozen shoulder (FS) is a painful restriction in both active and passive motion of the shoulder joint in all directions, including flexion, abduction and rotation ${ }^{1}$. Primary FS has high prevalence rate and long duration and can seriously affect the daily life and exercise regimen of patients ${ }^{2}$. FS is mainly associated with hyperthyroidism, hypothyroidism, diabetes, and Dupuytren disease $^{3}$. A large-scale prospective epidemiological study found that $1 \%$ of the population has FS, and women aged 40 to 60 years are more vulnerable than other populations ${ }^{4}$. Patients with FS describe the symptoms mainly as pain, which may be severe, not being able to sleep on the affected side, limited active and passive motion, and degenerated function. 
The pathogenesis of FS is not clear, and there has been no international standard definition ${ }^{5}$. Although FS is a self-limiting disease, it has a long course and leads to incomplete functional recovery ${ }^{6}$. Recent research has shown that this condition can lead to long-term disabilities ${ }^{7}$. Positive non-surgical and surgical treatment can shorten the course of the disease, accelerate recovery, help regain shoulder joint function and improve the quality of life of patients with $\mathrm{FS}^{8}$. Many studies have attempted to determine more effective treatments, but many controversies still exist. At present, there is no consensus for treating $\mathrm{FS}^{9}$. The lack of unified standards leads to complicated treatment options. Because FS is a very painful and disabling disease, appropriate treatment is especially important.

In recent years, with the continuous development and promotion of minimally invasive surgical techniques and arthroscopic techniques, the application of arthroscopic techniques to treat FS has gradually received increasing attention ${ }^{10,11,12}$. Shoulder arthroscopy has been widely accepted as a diagnostic and therapeutic tool for shoulder joint disease ${ }^{12}$. At present, many studies report that the main treatment for FS is arthroscopic capsule release, which is a safe and effective method ${ }^{13,14}$. The arthroscopic capsule release techniques are widely used and can be chosen depending on the surgeons' preference. In the past, some studies have noted that the inferior glenohumeral ligament (IGHL) plays an important role in maintaining the stability of the shoulder joint ${ }^{15,16}$.

At present, some senior doctors like to release the IGHL to treat FS, but no systematic literature has reported the effect of this approach on the stability and function of the shoulder joint ${ }^{11}$. The hypothesis of this prospective study is that arthroscopic capsule and IGHL release can improve the prognosis of FS and will not affect shoulder stability or cause dislocations in patients with FS. To prove this hypothesis, patients with FS were followed up for a mean of 28 weeks to further investigate the effect of arthroscopic capsule and IGHL release.

\section{Patients And Methods}

\section{Ethical support}

This was a prospective clinical trial study with 47 patients who underwent shoulder arthroscopic capsule and IGHL release performed by a senior doctor between January 2018 and June 2019. The study was approved by the Medical Ethics Committee of the Northern Jiangsu People's Hospital (number 2019029), and all 47 patients signed informed consent forms to participate in this study. The coordinating investigator will report any adverse events to the ethical committee in accordance with the ethical committee adverse events reporting procedures.

\section{Data collection and follow-up}

The data were collected at the Northern Jiangsu People's Hospital. A shoulder arthroscopic specialist team evaluated and informed all patients. Postoperative follow-up examinations were scheduled at 4, 8 , 
12 and 28 weeks at the orthopedics outpatient clinic of the study hospital. Three patients did not go to orthopedic outpatient clinics for review, and they were excluded at 12 weeks. Four patients did not go to orthopedic outpatient clinics for review, and they were excluded at 24 weeks. In total, 40 patients completed the 28-week follow-up, and the data of these patients were applied for statistical analyses and compared with the preoperative data. The experimental process is shown in Fig 1.

Among the 40 patients, there were 15 male patients and 25 female patients. There were 23 patients with left shoulder and 17 patients with right shoulder. There were 9 patients with diabetes and 31 patients without diabetes. The ages ranged from 41 to 77 years, and the mean age was $54.95 \pm 8.88$ years. The course of the disease ranged from 3 to 26 months, and the average disease duration was $7.18 \pm 5.98$ months.

\section{Inclusion and exclusion criteria}

Primary FS is not a definitive diagnostic criterion, and the diagnosis based on a physical examination ${ }^{17}$. All our studies are primary FS. A clinical diagnosis of FS can be made if the patient has painful, limited active and passive motion in at least two motion planes ${ }^{18}$. The inclusion criteria for this study were as follows: (1) restricted passive glenohumeral motion of $100^{\circ}$ or less during forward flexion (FF) and $20^{\circ}$ or less during external rotation (ER); (2) a history of at least 3 month of pain and stiffness; and (3) a normal radiologic appearance ${ }^{19}$. The exclusion criteria for this study were as follows: (1) acute shoulder injury; (2) combined with cervical spondylosis; (3) history of previous shoulder dislocation; (4) complicated cardiovascular and cerebrovascular diseases, nervous system diseases or blood system diseases; (5) combined with shoulder infections and tumors; (6) large and irreparable rotator cuff tears; and (7) combined with upper limb neuropathy.

\section{Functional assessments}

All of the patients were evaluated before and after surgery by the two professional orthopedists together completed a questionnaire with each patient about his or her subjective and objective symptoms. The primary outcome was the American Shoulder and Elbow Surgeons Score (ASES) score (scale 0-100, with higher scores indicating better outcomes and fewer symptoms). The secondary outcome was the Constant score criteria, which mainly accounts for subjective symptoms and the objective performance of the patients (scale 0-100, with higher scores indicating better outcomes and fewer symptoms). The third outcome was the visual analog scale (VAS) score for pain (range 0-10, 0 means no pain, and 10 means terrible pain). The fourth outcome was the active range of motion (ROM) ${ }^{20}$; the ROM includes FF and abduction ( $A B D)$, which were measured with a universal goniometer. The hand-behind-the-back method was used to measure internal rotation (IR). The hand-over-the-head method was used to measure ER. 
At 12 and 24 weeks, the apprehension test of the shoulder primarily checks for a possible torn labrum or anterior instability problems and was mainly applied to detect the stability of the arthroscopic capsule and IGHL release in the shoulder joint.

\section{Surgical procedures}

The patients who participated in this study all underwent the same surgical treatment. The affected shoulder was marked while the patient was under general anesthesia, and the patient was placed in the lateral position (Fig. 2A). Then, the affected shoulder joint was sterilized, and the area was draped (Fig. 2B). The surgeon first closed the manipulate to release the shoulder joint on the affected side. The affected shoulder was passively flexed and abducted to the maximum possible extent. Next, passive ER and IR were performed at $0^{\circ}$ of $A B D$ or $90^{\circ}$ of $A B D$.

A $30^{\circ}$ arthroscope (Smith \& Nephew, America) traversed a standard posterior portal between the head of the humerus and the biceps tendon. An anterior-superior portal was made just below the long head of the biceps tendon and positioned with a puncture needle under direct vision with the arthroscope (Fig. 3A). A radiofrequency probe was used to first release the rotator interval, then the superior capsule. At the same time, the superior glenohumeral ligament was dissected (Fig. 3B). The anterior capsular and middle glenohumeral ligaments of the affected shoulder were dissected through a rotator interval with a radiofrequency probe (Fig. $3 \mathrm{C}$ ). Then, the inferior capsular and anterior IGHL were dissected with the radiofrequency probe along the margin of the glenoid as far inferiorly as possible (Fig. 3D). To avoid surgically damaging the axillary nerve, a radiofrequency probe was inserted in the anterior-superior portal to dissect the inferior aspect of the capsule away from the axillary nerve. Finally, the residual posterior IGHL was dissected through the posterior portal.

\section{Postoperative rehabilitation}

All patients followed the same rehabilitation program at least once a day starting on postoperative day one; the program comprised shoulder ROM exercises, and exercises to strengthen the shoulder muscles were started as soon as the postoperative pain and active shoulder motion allowed. The patients were informed to perform each rehabilitation exercise twice a day. In weeks 1 to 4 , the rehabilitation exercises included passive FF and ABD with the patient in a supine position and passive ER and IR with the support of the unaffected side at $0^{\circ}$ of $A B D$. The patients were also encouraged to use the affected arm in activities of daily living. In weeks 5 to 8 , the affected shoulder joint gradually recovered the active ROM, and the patients actively increased the ROM of the affected shoulder joint. In weeks 9 to 12, exercises to strengthen the shoulder muscles of the affected side were performed. In weeks 13 to 28 , the patients independently performed these exercises at home.

\section{Statistics}


SPSS 20 software for Windows (SPSS Inc., Chicago, IL, USA) was used to record the data and for the statistical analyses. The data of the various measurements are presented as the means \pm standard deviations (SDs). Student's t-test was used to compare the 28-week follow-up data with the preoperative data. A p value $<0.05$ was considered statistically significant.

\section{Results}

\section{Apprehension test of the shoulder}

In this prospective study, 40 patients had negative apprehension tests at finally follow-up visits. No patients appeared to have shoulder joint instability or dislocations.

\section{Functional outcomes}

The mean ASES improved significantly from $31.30 \pm 6.41$ preoperatively to $92.43 \pm 3.89$ at the 28 -week follow-up. There were significantly different $(p<0.05)$. Similarly, the mean Constant score improved significantly from $30.15 \pm 6.85$ preoperatively to $90.71 \pm 4.27$ at the 28 -week follow-up. There were significantly different $(p<0.05)$. The mean VAS pain score also improved significantly from $6.73 \pm 0.72$ preoperatively to $0.60 \pm 0.74$ at the 28 -week follow-up. There were significantly different $(p<0.05)($ Table 1$)$.

Table 1

Changes in the preoperative and postoperative ASESs, Constant scores and VAS scores (scores, $X \pm S n=$ 40).

\begin{tabular}{|llll|}
\hline Time & ASES & Constant score & VAS score \\
\hline Preoperative & $31.30 \pm 6.41$ & $33.15 \pm 6.85$ & $6.73 \pm 0.72$ \\
\hline Week 4 & $43.40 \pm 7.54$ & $43.03 \pm 6.60$ & $4.03 \pm 0.70$ \\
\hline Week 8 & $58.35 \pm 5.02$ & $56.50 \pm 5.09$ & $2.63 \pm 0.70$ \\
\hline Week 12 & $81.93 \pm 4.21$ & $81.60 \pm 3.85$ & $1.35 \pm 0.80$ \\
\hline Week 28 & $92.43 \pm 3.89$ & $90.71 \pm 4.27$ & $0.60 \pm 0.74$ \\
\hline P value & 0.000 & 0.000 & 0.000 \\
\hline $\begin{array}{l}\text { The values are given as the mean and the standard deviation. ASES, American Shoulder and Elbow } \\
\text { Surgeons; VAS, visual analog scale. }\end{array}$
\end{tabular}

There were statistically significant differences in all ROM parameters between the final follow-up values and the preoperative values. The FF increased from $73.85 \pm 14.94$ to $166.70 \pm 7.23$, and the mean ABD increased from $69.65 \pm 12.74$ to $165.03 \pm 6.36$. The preoperative and postoperative ROM scores were significantly different $(p<0.05)($ Table 2$)$. 
Table 2

Preoperative and postoperative ROM flexion and abduction values $\left({ }^{\circ}, X \pm S n=40\right)$.

\begin{tabular}{|lll|}
\hline ROM & FF & ABD \\
\hline Preoperative & $73.85 \pm 14.94$ & $69.65 \pm 12.74$ \\
\hline Week 4 & $94.85 \pm 13.95$ & $88.75 \pm 13.22$ \\
\hline Week 8 & $125.60 \pm 14.21$ & $123.40 \pm 12.83$ \\
\hline Week 12 & $151.95 \pm 9.48$ & $154.48 \pm 6.44$ \\
\hline Week 28 & $166.70 \pm 7.23$ & $165.03 \pm 6.36$ \\
\hline P value & 0.000 & 0.000 \\
\hline $\begin{array}{l}\text { The values are given as the mean and standard deviation. Preoperative and postoperative active ROM } \\
\text { values. }\end{array}$ & \\
\hline
\end{tabular}

Preoperatively, no patients were able to perform full elevation of the arm or place the dorsum of their hands between their shoulder blades, showing limited ROMs. However, at the 28-week follow-up, $95 \%$ of the patients were observed to be able to perform full elevation, and $97.5 \%$ of the patients could place the dorsum of their hands between their shoulder blades (Table 3).

Table 3

Active ROM-preoperative and postoperative ER and IR values $(n=40)$

\begin{tabular}{|c|c|c|c|c|c|c|c|c|}
\hline & \multicolumn{8}{|l|}{ ROM } \\
\hline & \multicolumn{4}{|l|}{ ER } & \multicolumn{4}{|l|}{ IR } \\
\hline & $\begin{array}{l}\text { Hand } \\
\text { above } \\
\text { head and } \\
\text { elbow } \\
\text { forward }\end{array}$ & $\begin{array}{l}\text { Hand } \\
\text { above } \\
\text { head } \\
\text { and } \\
\text { elbow } \\
\text { back }\end{array}$ & $\begin{array}{l}\text { Full } \\
\text { elevation } \\
\text { of arm }\end{array}$ & Total & $\begin{array}{l}\text { Dorsum } \\
\text { the } \\
\text { hand to } \\
\text { the } \\
\text { waist }\end{array}$ & $\begin{array}{l}\text { Dorsum } \\
\text { of the } \\
\text { hand to } \\
\text { T } 12\end{array}$ & $\begin{array}{l}\text { Dorsuhm } \\
\text { the and } \\
\text { between } \\
\text { shoulder } \\
\text { blades }\end{array}$ & Total \\
\hline Preoperative & 36 & 4 & 0 & 40 & 35 & 5 & 0 & 40 \\
\hline Week 4 & 32 & 6 & 2 & 40 & 28 & 9 & 3 & 40 \\
\hline Week 8 & 18 & 16 & 6 & 40 & 16 & 17 & 7 & 40 \\
\hline Week 12 & 6 & 8 & 26 & 40 & 6 & 7 & 27 & 40 \\
\hline Week 28 & 0 & 2 & 38 & 40 & 0 & 1 & 39 & 40 \\
\hline
\end{tabular}

\section{Discussion}


FS is typically characterized by the spontaneous onset of pain with progressive, marked loss of active and passive shoulder ROM and stiffness at the glenohumeral joint, usually resulting in gross loss of function. Shaffer et al. ${ }^{21}$ have described that patients with FS treated nonoperatively still had slight pain or stiffness of the shoulder after 7 years. FS is considered difficult to treat, and there is no consensus regarding the optional management strategy ${ }^{17,22}$. The goals of treating FS are to relieve pain, restore shoulder joint function, and meet the needs daily life.

When patient conservative treatment fails, standardized surgical treatment approaches are particularly important. Recently Hagiwara et al. ${ }^{11}$ believed that arthroscopic coracohumeral ligament and capsular release could have excellent effects for patients with FS. Marina et al. ${ }^{14}$ published an article in 2014 and pointed out that arthroscopic capsular release alone or together with subacromial decompression was a safe procedure and showed favorable postoperative results. Some doctors have released the IGHL to improve the function of the FS but did not analyze whether this release would affect the function and stability of the shoulder joint ${ }^{11}$. We need to find a new procedure to quickly treat FS for patients early recover function. In our study, we mainly investigated the effect of IGHL release on function and stability in patients with FS.

FS could be addressed arthroscopically via the intra-articular approach, depending on the surgeon's preference. Either way, good visualization is undoubtedly the first consideration. The intra-articular approach allows for better visualization through a standard posterior viewing portal to allow for a clear observation of the thickening anterior joint capsule and the superior, middle and inferior glenohumeral ligament. The IGHL was divided into three parts: the axillary pouch, anterior bundle and posterior bundle $^{23}$. In our surgery, these parts were released. Inferior capsular and IGHL release should be performed near the glenoid labrum in the inferior pouch to minimize the risk of injury to the axillary nerve. Previous literature has reported that IGHL is in a state of tension when the shoulder joint is in ABD or $\mathrm{ER}^{24,25}$. Kim et al. ${ }^{26}$ and Bouaicha et al. ${ }^{27}$ indicated that when the shoulder $\mathrm{ABD}$ is greater than $45^{\circ}$, the axillary pouch and anterior bundle are important structures for maintaining stability in front of the shoulder joint. Although we paid close attention to anterior or posterior shoulder dislocations or apprehension during follow-up postoperative, none of the patients who underwent IGHL release experienced shoulder dislocations or apprehension. This indicated that IGHL release had no effect on the stability of the shoulder joint with FS. Why does the IGHL release procedure in the arthroscopic rotator cuff repair cannot cause instability of the patients with FS? Here set a reasonable inference that although patients going through the IGHL release, the adhesion reaction is still in proceed, which may cause the reconstruction of the ligament and capsule ${ }^{28}$. Therefore, it is not easy to cause instability.

According to some studies, the postoperative rehabilitation program may affect the prognosis of patients with $\mathrm{FS}^{29}$. A recent randomized controlled trial with level 1 evidence reported that a group exercise class provided superior outcomes in relieving the signs and symptoms of FS compared to other methods ${ }^{22}$. All patients in our study followed the same postoperative rehabilitation procedure, and we cannot conclude whether an accelerated rehabilitation procedure would affect recovering postoperative shoulder function. 
To objectively assess the impact of surgical interventions on FS, functional parameters may provide more objective results if validated scores are used. We used multiple shoulder function scores to ensure a high degree of accuracy. This prospective clinical trial showed that arthroscopic capsular and IGHL release was an effective intervention for patients with FS who still had severe pain and limited active and passive ROMs after conservative treatment. We found significant differences between the preoperative ASESs and Constant scores and those at the 28-week follow-up. Traditional arthroscopic capsular release without IGHL release is generally accepted and considered a safe surgical procedure ${ }^{30}$. This approach not only improves the ROM and the frequency and severity of the pain but also improves function ${ }^{31}$. The Constant scores of the current study and other relevant publications showed that arthroscopic treatment was an effective approach for FS. Compared to the studies from other authors, this study revealed higher Constant scores (Table 4). In our experiment, all ROM measures immediately improved postoperatively. The preoperative values of all ROM parameters were significantly different from those at the 28-week follow-up. We believe that arthroscopic capsular and IGHL release will have a tremendous effect during the short follow-up period, particularly in patients with FS who failed conservative treatment.

Table 4

Constant scores of this study (0-100 points) in comparison

with those of other studies (total cohort)

\begin{tabular}{|llll|}
\hline Authors & $\begin{array}{l}\text { Preoperative } \\
(\mathbf{0}-\mathbf{1 0 0})\end{array}$ & $\begin{array}{l}\text { Postoperative } \\
(\mathbf{0}-\mathbf{1 0 0})\end{array}$ & $\begin{array}{l}\text { Increase } \\
\text { in points }\end{array}$ \\
\hline J. Jerosch. $^{19}$ & 41 & 83 & 42 \\
\hline Gerber et al. $^{6}$ & 33 & 66 & 33 \\
\hline This trial & 33.15 & 90.71 & 57.56 \\
\hline
\end{tabular}

This study has several limitations. First, this study did not set up a control group that underwent only arthroscopic capsular release to compare and analyze the postoperative follow-up differences. Second, the sample size was small, and a large sample analysis should be performed in the future to determine the postoperative efficacy. Third, follow-up duration was short, and complications may occur during the long-term follow-up.

\section{Conclusion}

The current study provides new evidence regarding the choice of arthroscopic technique for FS. Our results suggest that this surgical procedure could significantly improve the function and active ROM of the affect shoulder and significantly reduce pain in patients with FS. Moreover, we did not observe any patients with shoulder instability or dislocations at any of the follow-up examinations. In fact, all patients achieved better active ROMs. 


\section{Abbreviations}

IGHL: inferior glenohumeral ligament; ASES: American Shoulder and Elbow Surgeons Score; VAS: visual analog scale; ROM: range of motion; FF: Forward flexion; ABD: abduction; ER: external rotation; IR: internal rotation.

\section{Declarations}

\section{Acknowledgements}

This study was approved by the Medical Ethics Committee of the Northern Jiangsu People's Hospital

\section{Funding}

No funding was received.

\section{Availability of data and materials}

The datasets generated and/or analyzed during the current study are not publicly available due the protection of the privacy of subjects involved and ongoing research but are available from the corresponding author on reasonable request.

\section{Author's contributions}

Zhang $Y$ conceived of the study and contributed to the critical revision of the article for important intellectual content. Cao SC drafted the manuscript and evaluated the data and performed statistical expertise and evaluated the data including collected and assembled the data. The Fei WY and Wang JC read and approved the final manuscript.

\section{Ethics approval and consent to participate}

The study was approved by the Medical Ethics Committee of the Northern Jiangsu People's Hospital (number 2019029). All patients provided informed consent to participate in this study.

\section{Consent for publication}

Not applicable.

\section{Competing interests}

The author declares that he has no competing interests.

\section{References}


1. Bunker TD, and Anthony PP. The pathology of frozen shoulder: a Dupuytren-like disease. Journal of Bone and Joint Surgery 1995; 5: 677-683.

2. Itoi E, Arce G, Bain GI, Diercks RL, Guttmann D, Imhoff AB. Shoulder Stiffness: Current Concepts and Concerns. Arthroscopy: The Journal of Arthroscopic \& Related Surgery 2016; 32: 1402-1414.

3. Holmes, RE, Barfield WR, and Woolf SK. Clinical evaluation of nonarthritic shoulder pain: Diagnosis and treatment. Phys Sportsmed 2015; 43: 262-268.

4. Huang YP, Fann CY, Chiu YH, Yen MF, Chen LS et al. Chen HH. Association of Diabetes Mellitus With the Risk of Developing Adhesive Capsulitis of the Shoulder: A Longitudinal Population-Based Followup Study. Arthritis Care \& Research 2013; 65: 1197-1202.

5. Kanazawa K, Hagiwara Y, Sekiguchi T, Suzuki K, Koide M, Akira A et al. Correlations between Capsular Changes and ROM Restriction in Frozen Shoulder Evaluated by Plain MRI and MR Arthrography. The Open Orthopaedics Journal 2018. 12(1): p. 396-404.

6. Jerosch $\mathrm{J} .360^{\circ}$ arthroscopic capsular release in patients with adhesive capsulitis of the glenohumeral joint-indication, surgical technique, results. Knee Surgery, Sports Traumatology, Arthroscopy 2001; 9: 178-186.

7. Hand C, Clipsham K, Rees JL, Carr AJ. Long-term outcome of frozen shoulder. Journal of Shoulder and Elbow Surgery 2008;17: 231-236.

8. Pearsall AW, and SpeerKP, Frozen shoulder syndrome: diagnostic and treatment strategies in the primary care setting. Med Sci Sports Exerc 1998; 30: 33-39.

9. Dundar U, Toktas H, Cakir T, Evcik D Kavuncuet V. Continuous passive motion provides good pain control in patients with adhesive capsulitis. International Journal of Rehabilitation Research 2009; 32: p. 193-198.

10. Gallacher S, Beazley JC, Evans J, Anaspure R, Silver D, Redfern A et al. A randomized controlled trial of arthroscopic capsular release versus hydrodilatation in the treatment of primary frozen shoulder. Journal of Shoulder and Elbow Surgery 2018; 27: 1401-1406.

11. Hagiwara Y, Ando A, Kanazawa K, Koide M, Sekiguchi T,Hamada J et al. Arthroscopic Coracohumeral Ligament Release for Patients With Frozen Shoulder. Arthroscopy Techniques 2018; 7: e1-e5.

12. Kanbe K. Clinical outcome of arthroscopic capsular release for frozen shoulder: essential technical points in 255 patients. Journal of orthopaedic surgery and research 2018. 13(1): p. 56-56.

13. Evans JP, Guyver PM, Smith CD. Frozen shoulder after simple arthroscopic shoulder procedures. Bone Joint J, 2015;97: 963-966.

14. Walther M, Blanke F, Wehren LV, Majewski M. Frozen shoulder-comparison of different surgical treatment options. Acta orthopaedica Belgica 2014; 80: 172-177.

15. 15.Lembeck B , Nikolaus Wülker. (i) Open procedures for shoulder instability[J]. Current Orthopaedics, 2004, 18:169-180.

16. Burkart A C, Debski R E . Anatomy and Function of the Glenohumeral Ligaments in Anterior Shoulder Instability. Clinical Orthopaedics and Related Research, 2002, 400:32-39. 
17. Lewis J. Frozen shoulder contracture syndrome-Aetiology, diagnosis and management. Manual Therapy 2015; 20: 2-9.

18. Buchbinder R, Hoving JL, Green S, Hall S, Forbes A, Nash P. Short course prednisolone for adhesive capsulitis (frozen shoulder or stiff painful shoulder): a randomised, double blind, placebo controlled trial. Annals of the Rheumatic Diseases, 2004. 63(11): p. 1460-1469.

19. Ando A, Sugaya H, Hagiwara $Y$, Takahashi N, Watanabe T, Kanazawa $K$ et al. Identification of prognostic factors for the nonoperative treatment of stiff shoulder. Int Orthop 2013; 37: 859-864.

20. Fieseler G, Molitor T, Irlenbusch L, Delank KS, Laudner KG, Hermassi S et al. Intrarater reliability of goniometry and hand-held dynamometry for shoulder and elbow examinations in female team handball athletes and asymptomatic volunteers. Archives of Orthopaedic and Trauma Surgery 2015; 135: 1719-1726.

21. Shaffer B, Tibone JE, Kerlan RK. Frozen Shoulder.A long-term follow-up. J Bone Joint Surg Am 1992; 74: 738-746.

22. Russell S, Jariwala A, Conlon R, Selfe J, Richards J, Walton M. A blinded, randomized, controlled trial assessing conservative management strategies for frozen shoulder. Journal of Shoulder and Elbow Surgery 2014; 23: 500-507.

23. Ticker JB, Flatow EL, Pawluk RJ, Soslowsky LJ, Ratcliffe A, Arnoczky SP et al. The inferior glenohumeral ligament: A correlative investigation. Journal of Shoulder and Elbow Surgery 2006; 15 : 665-674.

24. Shapiro TA, Gupta A, McGarry MH, Tibone JE, Lee TQ. Biomechanical Effects of Arthroscopic Capsulorrhaphy in Line With the Fibers of the Anterior Band of the Inferior Glenohumeral Ligament. The American Journal of Sports Medicine 2012; 40: 672-680.

25. 25.Ando A, Sugaya H, Takahashi N, Kawai N, Hagiwara Y, Itoi E. Arthroscopic Management of Selective Loss of External Rotation After Surgical Stabilization of Traumatic Anterior Glenohumeral Instability: Arthroscopic Restoration of Anterior Transverse Sliding Procedure. Arthroscopy: The Journal of Arthroscopic and Related Surgery, 2012. 28(6): p. 749-753.

26. Kim DW, Kim CK, Jung SW. An arthroscopic pleated capsular shift for recurrent anterior dislocation of the shoulder. Knee Surgery, Sports Traumatology, Arthroscopy 2012; 20: 2579-2584.

27. 27.Bouaicha S, Moor BK. Arthroscopic autograft reconstruction of the inferior glenohumeral ligament: Exploration of technical feasibility in cadaveric shoulder specimens. International Journal of Shoulder Surgery 2013; 7: 32.

28. Park JH, Yang SH, Rhee SM, Oh JH. The effect of concomitant coracohumeral ligament release in arthroscopic rotator cuff repair to prevent postoperative stiffness: a retrospective comparative study. Knee Surgery, Sports Traumatology, Arthroscopy 2019;

29. Dupeyron A, De'narie M', Richard D, Dobija L, Castelli C, Petiot S et al. Analgesic gas for rehabilitation of frozen shoulder: Protocol for a randomized controlled trial. Annals of Physical and Rehabilitation Medicine 2019; 62: 43-48. 
30. Hagiwara Y, Sugaya H, Takahashi N, Kawai N, Ando A, Hamada J et al. Effects of intra-articular steroid injection before pan-capsular release in patients with refractory frozen shoulder. Knee Surgery, Sports Traumatology, Arthroscopy 2015; 23: 1536-1541.

31. Jerosch J, Nasef NM, OPeters O, Reda Mansour AM. Mid-term results following arthroscopic capsular release in patients with primary and secondary adhesive shoulder capsulitis. Knee Surgery, Sports Traumatology, Art 2013;5:1195-1202.

32. Castellarin G, Ricci M,VedoviE, Vecchini E, Sembenini P, Marangon A et al. Manipulation and arthroscopy under general anesthesia and early rehabilitative treatment for frozen shoulders. Arch Phys Med Rehabil 2004; 85: 1236-1240.

\section{Figures}

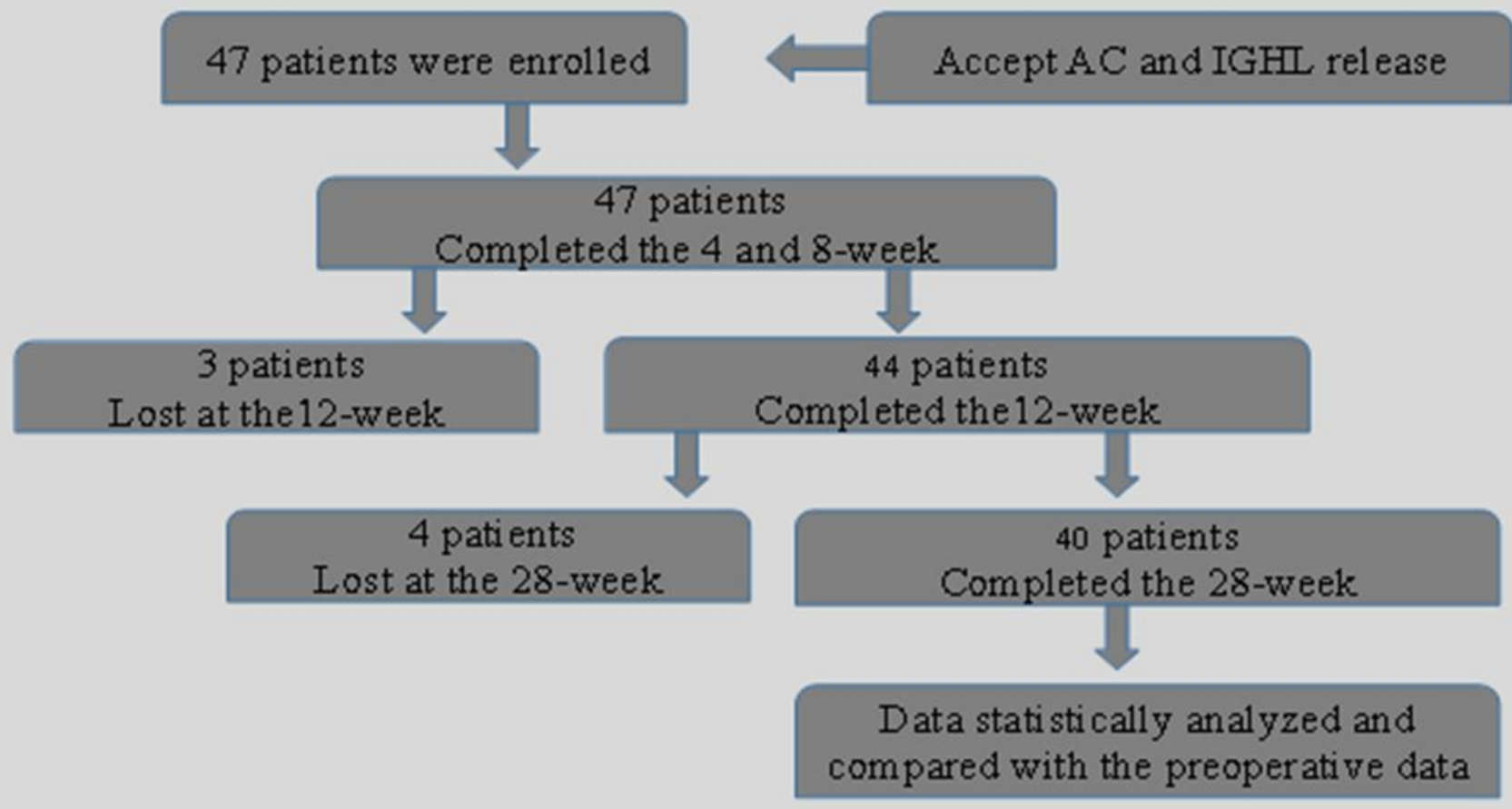

\section{Figure 1}

The experimental process. 

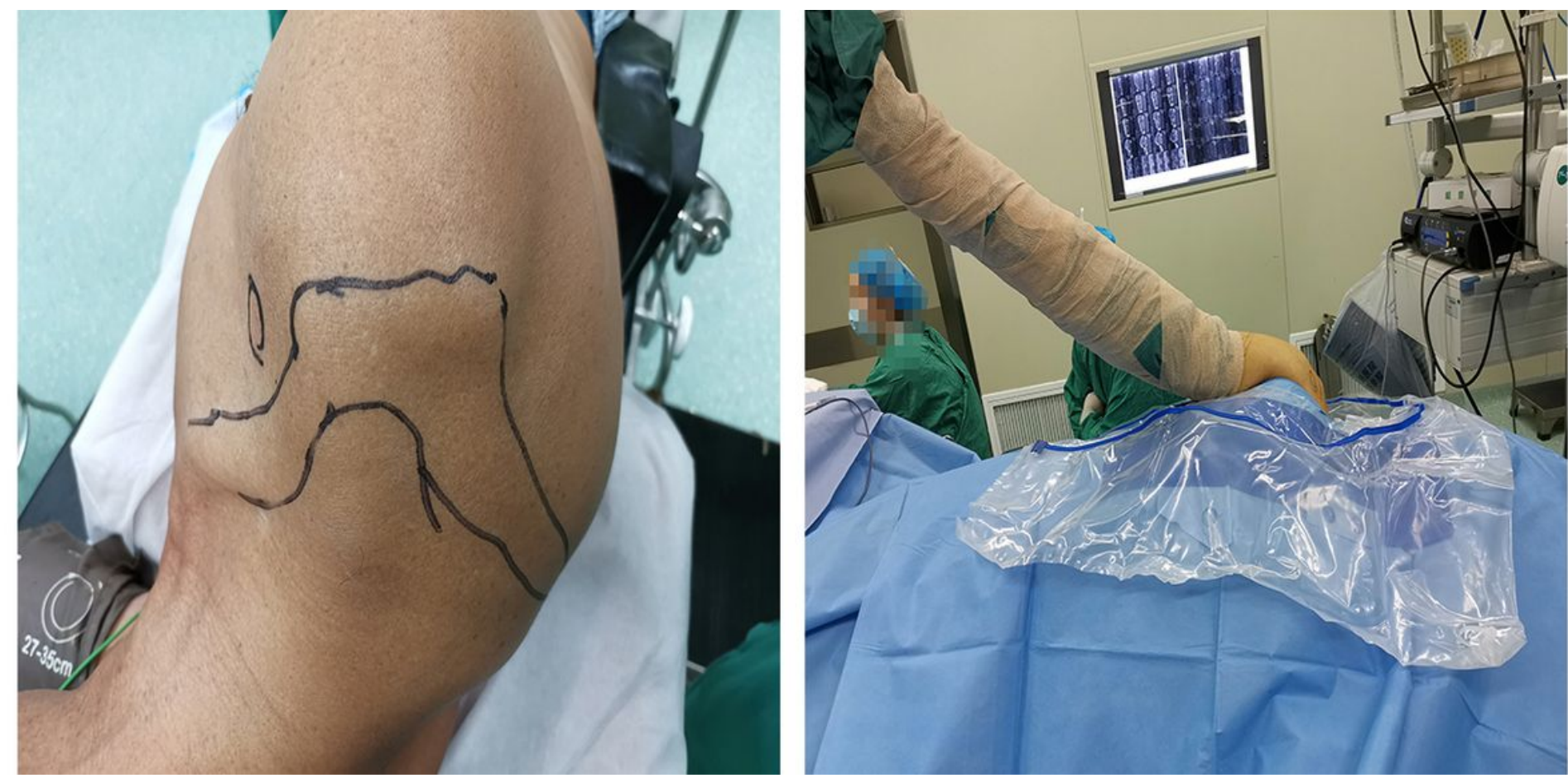

Figure 2

The affected shoulder was marked while the patient was under general anesthesia, and the patient was placed in the lateral position (Fig. 2A). Then, the affected shoulder joint was sterilized, and the area was draped (Fig. 2B).
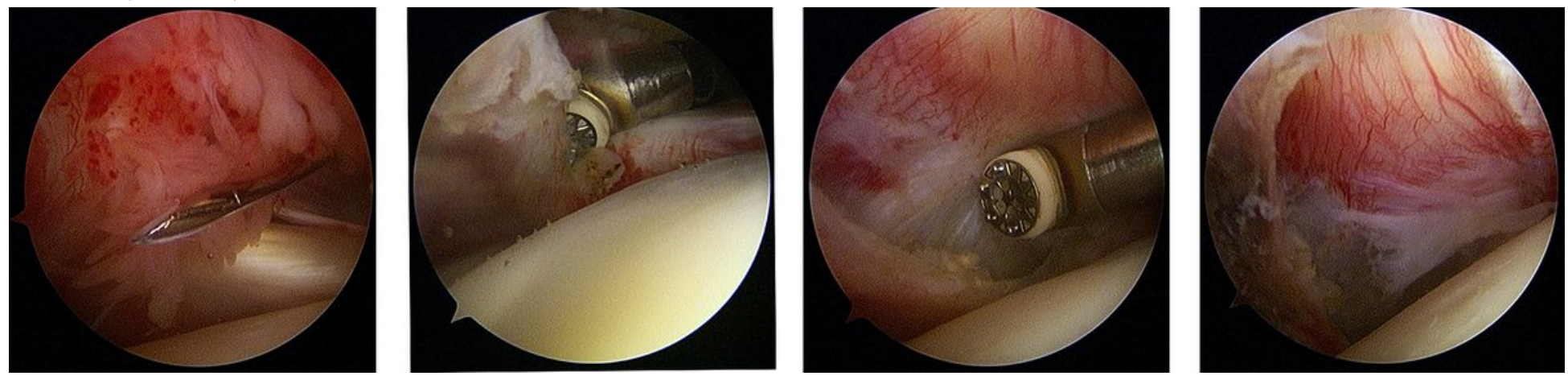

\section{Figure 3}

An anterior-superior portal was made just below the long head of the biceps tendon and positioned with a puncture needle under direct vision with the arthroscope (Fig. 3A). A radiofrequency probe was used to first release the rotator interval, then the superior capsule. At the same time, the superior glenohumeral ligament was dissected (Fig. 3B). The anterior capsular and middle glenohumeral ligaments of the affected shoulder were dissected through a rotator interval with a radiofrequency probe (Fig. $3 \mathrm{C}$ ). Then, the inferior capsular and anterior IGHL were dissected with the radiofrequency probe along the margin of the glenoid as far inferiorly as possible (Fig. 3D). 